\title{
Lebesgue Decomposition Theorem and Weak Radon-Nikodým Theorem for Generalized Fuzzy Number Measures
}

\author{
Cai-Li Zhou' ${ }^{1}$ and Fu-Gui Shi \\ ${ }^{1}$ College of Mathematics and Information Science, Hebei University, Baoding 071002, China \\ ${ }^{2}$ School of Mathematics and Statistics, Beijing Institute of Technology, Beijing 100081, China
}

Correspondence should be addressed to Cai-Li Zhou; hyyanyiyi@163.com

Received 17 September 2015; Accepted 27 October 2015

Academic Editor: Luisa Di Piazza

Copyright (C) 2015 C.-L. Zhou and F.-G. Shi. This is an open access article distributed under the Creative Commons Attribution License, which permits unrestricted use, distribution, and reproduction in any medium, provided the original work is properly cited.

The Lebesgue type decomposition theorem and weak Radon-Nikodým theorem for fuzzy valued measures in separable Banach spaces are established.

\section{Introduction}

The topic of set valued and fuzzy valued measures has received much attention in the past few decades because of its usefulness in several applied fields like mathematical economics and optimal control. Significant contributions to set valued measures were made by Artstein [1], Cascales et al. [2, 3], Hiai [4], Papageorgiou [5], Stojaković [6], Zhang et al. [7], Zhou and Shi [8], and others. Fuzzy valued measure is a natural generalization of set valued measures. Hence the study of fuzzy valued measures is usually connected with set valued measures. Contributions in this field were made, among others, by Puri and Ralescu [9], Bán [10], Stojaković $[11,12]$, M. Stojaković and Z. Stojaković [13, 14], Xue et al. [15], and Park [16].

The main results of this paper fall into two main parts. It is well known that Lebesgue type decomposition theorem and Radon-Nikodým theorem are very important results in measure theory. As an extension of Lebesgue type decomposition theorem for vector measures, Zhang et al. [7] obtained Lebesgue type decomposition theorem for set valued measures. In the first part we generalize these results to generalized fuzzy number measures. On the other hand, Wu et al. [17] obtained Radon-Nikodým theorem for generalized fuzzy number measures using Bochner integral. But it is well known that Pettis integrability is a more general concept than that of Bochner integrability in the theory of integration in infinite dimensional spaces. In the second part, we obtain weak Radon-Nikodým theorem for generalized fuzzy number measures using Pettis type integral.

The paper is structured as follows. In Section 2, we state some basic concepts and preliminary results. In Section 3, the Lebesgue type decomposition theorem and weak RadonNikodým theorem for generalized fuzzy number measures will be established.

\section{Preliminaries}

Throughout this paper, let $(\Omega, \mathscr{A}, \mu)$ be a complete finite measure space, where $\Omega$ is a nonempty set, $\mathscr{A}$ is a $\sigma$-algebra of subsets of $\Omega$, and $\mu$ is a measure. Let $(\mathscr{X},\|\cdot\|)$ be a real separable Banach space with its dual space $\mathscr{X}^{*}$. Let

$$
\begin{aligned}
& \mathscr{P}_{0}(\mathscr{X})=\{A \subset \mathscr{X}: A \text { is a nonempty subset of } \mathscr{X}\}, \\
& \mathscr{P}_{(f)(c)}(\mathscr{X})=\left\{A \in \mathscr{P}_{0}(\mathscr{X}): A\right. \text { is bounded } \\
& \text { (closed) (convex) }\}, \\
& \mathscr{P}_{w k c}(\mathscr{X})=\left\{A \in \mathscr{P}_{0}(\mathscr{X}): A\right. \text { is weakly compact and } \\
& \text { convex }\} .
\end{aligned}
$$

For $A, B \in \mathscr{P}_{f}(\mathscr{X})$, the Hausdorff metric $d_{H}$ of $A$ and $B$ is defined by

$$
d_{H}(A, B)=\max \left\{\sup _{x \in A} \inf _{y \in B}\|x-y\|, \sup _{y \in B} \inf _{x \in A}\|x-y\|\right\} .
$$


Note that $\left(\mathscr{P}_{w k c}(\mathscr{X}), d_{H}\right)$ is a complete metric space. The number $|A|$ is defined by $|A|=d_{H}(A,\{0\})=\sup _{x \in A}\|x\|$ for each $A \in \mathscr{P}_{f}(\mathscr{X})$.

We will denote by $\sigma(\cdot, A)$ the support function of a set $A \subset$ $\mathscr{X}$ defined by

$$
\sigma\left(x^{*}, A\right)=\sup _{x \in A}\left\langle x^{*}, x\right\rangle, \quad x^{*} \in \mathscr{X}^{*} .
$$

The support function satisfies the following properties: $\sigma\left(x^{*}, A+B\right)=\sigma\left(x^{*}, A\right)+\sigma\left(x^{*}, B\right)$ and $\sigma\left(x^{*}, \lambda A\right)=\lambda \sigma\left(x^{*}, A\right)$ for all $A, B \in \mathscr{P}_{0}(\mathscr{X})$ and $\lambda \geq 0$.

Definition 1 (see [15]). Let $\tilde{u}: \mathscr{X} \rightarrow[0,1]$. One denotes $\tilde{u}_{\alpha}=\{x \in \mathscr{X}: \widetilde{u}(x) \geq \alpha\}$ for each $\alpha \in(0,1] . \tilde{u}$ is called a generalized fuzzy number if, for each $\alpha \in(0,1]$, $\widetilde{u}_{\alpha} \in \mathscr{P}_{w k c}(\mathscr{X})$. Let $\mathscr{F}_{w k c}(\mathscr{X})$ denote the set of all generalized fuzzy numbers on $\mathscr{X}$.

For $\widetilde{u}, \widetilde{v} \in \mathscr{F}_{w k c}(\mathscr{X})$, we define $\widetilde{u}+\widetilde{v}$ as follows:

$$
(\widetilde{u}+\widetilde{v})(x)=\sup _{x=y+z} \min \{\tilde{u}(y), \widetilde{v}(z)\}
$$

Obviously, we have $(\tilde{u}+\widetilde{v})_{\alpha}=\widetilde{u}_{\alpha}+\widetilde{v}_{\alpha}$ for each $\alpha \in(0,1]$, and therefore $\widetilde{u}+\widetilde{v} \in \mathscr{F}_{w k c}(\mathscr{X})$. In the set $\mathscr{F}_{w k c}(\mathscr{X})$ we define $d_{H}^{\infty}$ by

$$
d_{H}^{\infty}(\widetilde{u}, \widetilde{v})=\sup _{\alpha \in(0,1]} d_{H}\left(\widetilde{u}_{\alpha}, \widetilde{v}_{\alpha}\right)
$$

$\left(\mathscr{F}_{w k c}(\mathscr{X}), d_{H}^{\infty}\right)$ is a metric space.

Theorem 2 (see [18]). If $\tilde{u} \in \mathscr{F}_{w k c}(\mathscr{X})$, then one has the following:

(1) $\tilde{u}_{\alpha} \in \mathscr{P}_{w k c}(\mathscr{X})$ for all $\alpha \in(0,1]$.

(2) $\tilde{u}_{\alpha} \supseteq \tilde{u}_{\beta}$ for $0<\alpha \leq \beta \leq 1$.

(3) If $\left\{\alpha_{n}\right\}_{n \in \mathbb{N}}$ is a nondecreasing sequence in $[0,1]$ converging to $\alpha \in(0,1]$, then $\widetilde{u}_{\alpha}=\bigcap_{n=1}^{\infty} \widetilde{u}_{\alpha_{n}}$.

Conversely, if $\left\{A_{\alpha}: \alpha \in(0,1]\right\} \subseteq \mathscr{P}_{0}(\mathscr{X})$ satisfies (1)-(3) above, then there exists a $\tilde{u} \in \mathscr{F}_{w k c}(\mathscr{X})$ such that $\tilde{u}_{\alpha}=A_{\alpha}$ for each $\alpha \in(0,1]$.

Theorem 3 (see [19]). Let $M_{\alpha} \in \mathscr{P}_{w k c}(\mathscr{X}),\left\{M_{\alpha_{n}}\right\}_{n \in \mathbb{N}} \subset$ $\mathscr{P}_{w k c}(\mathscr{X})$, and $\alpha_{n} \nearrow \alpha, M_{\alpha_{n}} \supset M_{\alpha_{n+1}} \supset M_{\alpha}$; then $\sigma\left(x^{*}, M_{\alpha_{n}}\right)$ converges to $\sigma\left(x^{*}, M_{\alpha}\right)$ for each $x^{*} \in \mathscr{X}^{*}$ if and only if $M_{\alpha}=$ $\bigcap_{n=1}^{\infty} M_{\alpha_{n}}$.

Definition 4 (see [4]). Let $(\Omega, \mathscr{A})$ be a measurable space. The mapping $\mathscr{M}: \mathscr{A} \rightarrow \mathscr{P}_{0}(\mathscr{X})$ is said to be a set valued measure if it satisfies the following two conditions:

(1) $\mathscr{M}(\emptyset)=\{0\}$;
(2) if $A_{1}, A_{2}, \ldots$ are in $\mathscr{A}$, with $A_{i} \cap A_{j}=\emptyset$ for $i \neq j$, then

$$
\mathscr{M}\left(\bigcup_{i=1}^{\infty} A_{i}\right)=\sum_{i=1}^{\infty} \mathscr{M}\left(A_{i}\right)
$$

where $\sum_{i=1}^{\infty} \mathscr{M}\left(A_{i}\right)=\{x \in \mathscr{X}: x=$ $\sum_{n=1}^{\infty} x_{i}$ (unc.conv.), $\left.x_{i} \in \mathscr{M}\left(A_{i}\right), i \geq 1\right\}$.

Particularly, $\mathscr{M}: \mathscr{A} \rightarrow \mathscr{P}_{w k c}(\mathscr{X})$ is a set valued measure if and only if $\sigma\left(x^{*}, \mathscr{M}(\cdot)\right)$ is a real valued measure for all $x^{*} \in$ $x^{*}$.

As for single valued measures, we have the notion of total variation $|\mathscr{M}|$ of $\mathscr{M}$. For $A \in \mathscr{A}$ we define $|\mathscr{M}|(A)=$ $\sup \sum_{i=1}^{n}\left|\mathscr{M}\left(A_{i}\right)\right|$, where the supremum is taken over all finite measurable partitions $\left\{A_{1}, \ldots, A_{n}\right\}$ of $A$. We call that $\mathscr{M}$ is of bounded variation if $|\mathscr{M}|(\Omega)<\infty$. We call that $\mathscr{M}$ is of $\sigma$-bounded variation if there exists a countably measurable partition $\left\{A_{n}\right\}_{n \in \mathbb{N}}$ such that each restriction of $\mathscr{M}$ to $A_{n}$ is of bounded variation. We call that $\mathscr{M}$ is continuous absolutely about $\mu$ if, for any $A \in \mathscr{A}, \mu(A)=0$; then $\mathscr{M}(A)=\{0\}$, denoted as $\mathscr{M} \ll \mu$. We call that $\mathscr{M}$ is singular about $\mu$ if there exists $N \in \mathscr{A}, \mu(N)=0$, such that, for any $A \in \mathscr{A}$, $\mathscr{M}\left(A \cap N^{c}\right)=\{0\}$, denoted as $\mathscr{M} \perp \mu$.

Definition 5 (see [15]). Let $(\Omega, \mathscr{A})$ be a measurable space. The mapping $\widetilde{\mathscr{M}}: \mathscr{A} \rightarrow \mathscr{F}_{w k \mathrm{c}}(\mathscr{X})$ is called a generalized fuzzy number measure if it satisfies the following two conditions:

(1) $\widetilde{\mathscr{M}}(\emptyset)=\widetilde{0}$, where $\widetilde{0}$ is indicator function of $\{0\}$.

(2) If $A_{1}, A_{2}, \ldots$ are in $\mathscr{A}$, with $A_{i} \cap A_{j}=\emptyset$ for $i \neq j$, then

$$
\widetilde{\mathscr{M}}\left(\bigcup_{i=1}^{\infty} A_{i}\right)=\sum_{i=1}^{\infty} \widetilde{\mathscr{M}}\left(A_{i}\right)
$$

$$
\text { where }\left(\sum_{i=1}^{\infty} \widetilde{\mathscr{M}}\left(A_{i}\right)\right)(x)=\sup \left\{\bigwedge_{i=1}^{\infty} \widetilde{\mathscr{M}}\left(A_{i}\right)\left(x_{i}\right): x=\right.
$$
$\sum_{i=1}^{\infty} x_{i}$ (unc.conv.) $\}$.

We call that $\widetilde{\mathscr{M}}$ is continuous absolutely about $\mu$ if, for any $A \in \mathscr{A}, \mu(A)=0$; then $\widetilde{\mathscr{M}}(A)=\widetilde{0}$, denoted as $\widetilde{\mathscr{M}} \ll \mu$.

Theorem 6 (see [15]). The mapping $\widetilde{\mathscr{M}}: \mathscr{A} \rightarrow \mathscr{F}_{w k c}(\mathscr{X})$ is a generalized fuzzy number measure if and only if there exists a family of set valued measures $\mathscr{M}_{\alpha}: \mathscr{A} \rightarrow \mathscr{P}_{w k c}(\mathscr{X}), \alpha \in(0,1]$ satisfying the following three conditions:

(1) For arbitrary $\alpha, \beta \in(0,1]$ and $A \in \mathscr{A}$, if $\alpha \leq \beta$, then $\mathscr{M}_{\alpha}(A) \supseteq \mathscr{M}_{\beta}(A)$.

(2) For arbitrary $\left\{\alpha_{n}\right\}_{n \in \mathbb{N}} \subseteq(0,1]$ and $\alpha \in(0,1]$ such that $\alpha_{1} \leq \alpha_{2} \leq \cdots$ and $\lim _{n \rightarrow \infty} \alpha_{n}=\alpha$, then $\mathscr{M}_{\alpha}(A)=$ $\bigcap_{n=1}^{\infty} \mathscr{M}_{\alpha_{n}}(A), \forall A \in \mathscr{A}$.

(3) For arbitrary $A \in \mathscr{A}$, one has

$$
\widetilde{M}(A)(x)= \begin{cases}\sup \left\{\alpha: x \in \mathscr{M}_{\alpha}(A), \alpha \in(0,1]\right\}, & \text { if }\left\{\alpha: x \in \mathscr{M}_{\alpha}(A), \alpha \in(0,1]\right\} \neq \emptyset \\ 0, & \text { if }\left\{\alpha: x \in \mathscr{M}_{\alpha}(A), \alpha \in(0,1]\right\}=\emptyset\end{cases}
$$


Note that for a generalized fuzzy number measure $\widetilde{\mathscr{M}}$ : $\mathscr{A} \rightarrow \mathscr{F}_{w k c}(\mathscr{X})$ the set valued measure $\mathscr{M}_{\alpha}: \mathscr{A} \rightarrow \mathscr{P}_{w k c}(\mathscr{X})$ is determined by

$$
\mathscr{M}_{\alpha}(A)=\{x \in \mathscr{X}: \widetilde{\mathscr{M}}(A)(x) \geq \alpha\} ;
$$

that is, $\mathscr{M}_{\alpha}(A)=[\widetilde{\mathscr{M}}(A)]_{\alpha}$.

\section{Main Results}

In this section, we first give the Lebesgue type decomposition theorem for generalized fuzzy number measures. Our result is a generalization of Zhang et al's result [7]. And then, we obtain weak Radon-Nikodým theorem for generalized fuzzy number measures.

Theorem 7 (see [7] Lebesgue type decomposition theorem for set valued measures). Let $\mathscr{M}: \mathscr{A} \rightarrow \mathscr{P}_{\text {wkc }}(\mathscr{X})$ be a set valued measure. Then there exists a unique pair of weakly compact and convex set valued measures $\mathscr{M}_{c}$ and $\mathscr{M}_{s}$ such that

(1) $\mathscr{M}_{c} \ll \mu, \mathscr{M}_{s} \perp \mu$;

(2) $\mathscr{M}=\mathscr{M}_{c}+\mathscr{M}_{s}$;

(3) for arbitrary $x^{*} \in \mathscr{X}^{*}$,

$$
\sigma\left(x^{*}, \mathscr{M}(\cdot)\right)=\sigma\left(x^{*}, \mathscr{M}_{c}(\cdot)\right)+\sigma\left(x^{*}, \mathscr{M}_{s}(\cdot)\right)
$$

is the Lebesgue decomposition of $\sigma\left(x^{*}, \mathscr{M}(\cdot)\right)$.

In what follows, we first give the definition of singular for generalized fuzzy number measure about crisp measure and give two lemmas before we establish the main result.

Definition 8 . Let $(\Omega, \mathscr{A}, \mu)$ be a finite real valued measure space and let $\widetilde{\mathscr{M}}: \mathscr{A} \rightarrow \mathscr{F}_{w k c}(\mathscr{X})$ be a generalized fuzzy number measure. One calls that $\widetilde{\mathscr{M}}$ is singular about $\mu$ if there exists $N \in \mathscr{A}, \mu(N)=0$, such that, for any $A \in \mathscr{A}$, $\widetilde{\mathscr{M}}\left(A \cap N^{c}\right)=\widetilde{0}$, denoted as $\widetilde{\mathscr{M}} \perp \mu$.

Lemma 9. Let $(\Omega, \mathscr{A}, \mu)$ be a finite real valued measure space and let $\widetilde{\mathscr{M}}: \mathscr{A} \rightarrow \mathscr{F}_{w k c}(\mathscr{X})$ be a generalized fuzzy number measure. Then

(1) $\widetilde{\mathscr{M}} \ll \mu$ if and only if $\widetilde{\mathscr{M}}_{\alpha} \ll \mu$ for all $\alpha \in(0,1]$;

(2) $\widetilde{\mathscr{M}} \perp \mu$ if and only if $\widetilde{\mathscr{M}}_{\alpha} \perp \mu$ for all $\alpha \in(0,1]$.

Proof. (1) Suppose that $\widetilde{\mathscr{M}} \ll \mu$. For any $A \in \mathscr{A}$, if $\mu(A)=0$ then $\widetilde{\mathscr{M}}(A)=\widetilde{0}$, which implies that $\widetilde{\mathscr{M}}_{\alpha}(A)=\{0\}$ for all $\alpha \in$ $(0,1]$; that is, $\widetilde{\mathscr{M}}_{\alpha} \ll \mu$ for all $\alpha \in(0,1]$.

On the contrary, suppose that $\widetilde{\mathscr{M}}_{\alpha} \ll \mu$ for all $\alpha \in(0,1]$. For any $A \in \mathscr{A}$, if $\mu(A)=0$ then $\widetilde{\mathscr{M}}_{\alpha}(A)=\{0\}$. If $\widetilde{\mathscr{M}}(A) \neq$ $\widetilde{0}$, then there exists $x \in \mathcal{X}$ such that $\widetilde{\mathscr{M}}(A)(x)>0$, which leads to the conclusion that there exists $\alpha \in(0,1]$ such that $x \in \widetilde{\mathscr{M}}_{\alpha}(A)$. This contradicts the fact that $\widetilde{\mathscr{M}}_{\alpha}(A)=\{0\}$ for all $\alpha \in(0,1]$. Hence, $\widetilde{\mathscr{M}}(A)=\widetilde{0}$. This implies that $\widetilde{\mathscr{M}} \ll \mu$.

(2) Suppose that $\widetilde{\mathscr{M}} \perp \mu$. If there exists $N \in \mathscr{A}, \mu(N)=0$, then, for any $A \in \mathscr{A}, \widetilde{M}\left(A \cap N^{c}\right)=\widetilde{0}$ which implies that
$\widetilde{\mathscr{M}}_{\alpha}\left(A \cap N^{c}\right)=\{0\}$ for all $\alpha \in(0,1]$. It follows that $\widetilde{\mathscr{M}}_{\alpha} \perp \mu$ for all $\alpha \in(0,1]$.

Conversely, suppose that $\widetilde{\mathbb{M}}_{\alpha} \perp \mu$ for all $\alpha \in(0,1]$. According to Definition 8, if there exists $N \in \mathscr{A}, \mu(N)=0$, then, for any $A \in \mathscr{A}$ and $\alpha \in(0,1]$, we have $\widetilde{\mathscr{M}}_{\alpha}\left(A \cap N^{c}\right)=\{0\}$, which implies that $\widetilde{\mathscr{M}}\left(A \cap N^{c}\right)=\widetilde{0}$. Hence, $\widetilde{\mathscr{M}} \perp \mu$.

Lemma 10. Let $\left\{\alpha_{n}\right\}_{n \in \mathbb{N}} \subseteq(0,1]$ such that $\alpha_{n} \nearrow \alpha$ and let $\left\{\mathscr{M}_{\alpha_{n}}(\cdot)\right\}_{n \in \mathbb{N}}$ be a family of weakly compact and convex set valued measure such that $\mathscr{M}_{\alpha}(A) \subset \mathscr{M}_{\alpha_{n+1}}(A) \subset \mathscr{M}_{\alpha_{n}}(A)$ for any $A \in \mathscr{A}$. Then one has the following:

(1) $\lim _{n \rightarrow \infty} \sigma\left(x^{*}, \mathscr{M}_{\alpha_{n}}(\cdot)\right)$ is a real valued measure for each $x^{*} \in \mathscr{X}^{*}$.

(2) If $\mathscr{M}_{\alpha_{n}} \ll \mu$ for each $\alpha_{n} \in(0,1]$, then $\lim _{n \rightarrow \infty} \sigma\left(x^{*}, \mathscr{M}_{\alpha_{n}}(\cdot)\right) \ll \mu$ for each $x^{*} \in \mathscr{X}^{*}$.

(3) If $\mathscr{M}_{\alpha_{n}} \perp \mu$ for each $\alpha_{n} \in(0,1]$, then $\lim _{n \rightarrow \infty} \sigma\left(x^{*}, \mathscr{M}_{\alpha_{n}}(\cdot)\right) \perp \mu$ for each $x^{*} \in \mathscr{X}^{*}$.

Proof. (1) Since $\mathscr{M}_{\alpha_{n}}: \Omega \rightarrow \mathscr{P}_{w k c}(\mathscr{X})$ is a set valued measure for any $n \in \mathbb{N}, \sigma\left(x^{*}, \mathscr{M}_{\alpha_{n}}(\cdot)\right)$ is a real valued measure for each $x^{*} \in \mathscr{X}^{*}$ and any $n \in \mathbb{N}$. Further,

$$
\mathscr{M}_{\alpha}(A) \subset \cdots \subset \mathscr{M}_{\alpha_{n}}(A) \subset \cdots \subset \mathscr{M}_{\alpha_{1}}(A)
$$

implies

$$
\begin{aligned}
\sigma\left(x^{*}, \mathscr{M}_{\alpha}(A)\right) & \leq \cdots \leq \sigma\left(x^{*}, \mathscr{M}_{\alpha_{n}}(A)\right) \leq \cdots \\
& \leq \sigma\left(x^{*}, \mathscr{M}_{\alpha_{1}}(A)\right)
\end{aligned}
$$

for each $x^{*} \in \mathscr{X}^{*}$ and $A \in \mathscr{A}$, which shows that $\left\{\sigma\left(x^{*}, \mathscr{M}_{\alpha_{n}}(A)\right)\right\}_{n \in \mathbb{N}}$ is a monotone decreasing and bounded sequence for each $x^{*} \in \mathscr{X}^{*}$ and $A \in \mathscr{A}$. This ensures that $\lim _{n \rightarrow \infty} \sigma\left(x^{*}, \mathscr{M}_{\alpha_{n}}(A)\right)$ exists. In the following, we claim that $\lim _{n \rightarrow \infty} \sigma\left(x^{*}, \mathscr{M}_{\alpha_{n}}(\cdot)\right)$ is a real valued measure for each $x^{*} \in$ $X^{*}$. Obviously, $\lim _{n \rightarrow \infty} \sigma\left(x^{*}, \mathscr{M}_{\alpha_{n}}(\emptyset)\right)=0$. Let $\left\{A_{j}\right\}_{j \in \mathbb{N}}$ be a sequence of pairwise disjoint elements of $\mathscr{A}$. By countable additivity of set valued measure and Theorem 6.1.1 [7], we have

$$
\begin{aligned}
& \lim _{n \rightarrow \infty} \sigma\left(x^{*}, \mathscr{M}_{\alpha_{n}}\left(\bigcup_{j=1}^{\infty} A_{j}\right)\right) \\
& =\lim _{n \rightarrow \infty} \sigma\left(x^{*}, \sum_{j=1}^{\infty} \mathscr{M}_{\alpha_{n}}\left(A_{j}\right)\right) \\
& =\lim _{n \rightarrow \infty} \sum_{j=1}^{\infty} \sigma\left(x^{*}, \mathscr{M}_{\alpha_{n}}\left(A_{j}\right)\right)
\end{aligned}
$$


for each $x^{*} \in \mathscr{X}^{*}$. Sine for each $A_{j} \in \mathscr{A}$ and $\alpha_{n} \in(0,1]$, we have

$$
\begin{aligned}
\left|\sigma\left(x^{*}, \mathscr{M}_{\alpha_{n}}\left(A_{j}\right)\right)\right| & \leq\left\|x^{*}\right\|\left|\mathscr{M}_{\alpha_{n}}\left(A_{j}\right)\right| \\
& \leq\left\|x^{*}\right\|\left|\mathscr{M}_{\alpha_{1}}\left(A_{j}\right)\right| \\
& \leq\left\|x^{*}\right\|\left|\mathscr{M}_{\alpha_{1}}\right|\left(A_{j}\right), \\
\sum_{j=1}^{\infty}\left\|x^{*}\right\|\left|\mathscr{M}_{\alpha_{1}}\right|\left(A_{j}\right) & =\left\|x^{*}\right\| \sum_{j=1}^{\infty}\left|\mathscr{M}_{\alpha_{1}}\right|\left(A_{j}\right) \\
& =\left\|x^{*}\right\|\left|\mathscr{M}_{\alpha_{1}}\right|\left(\bigcup_{j=1}^{\infty} A_{j}\right)<\infty,
\end{aligned}
$$

which implies that

$$
\begin{array}{r}
\lim _{n \rightarrow \infty} \sigma\left(x^{*}, \mathscr{M}_{\alpha_{n}}\left(\bigcup_{j=1}^{\infty} A_{j}\right)\right) \\
=\lim _{n \rightarrow \infty} \sum_{j=1}^{\infty} \sigma\left(x^{*}, \mathscr{M}_{\alpha_{n}}\left(A_{j}\right)\right) \\
=\sum_{j=1}^{\infty} \lim _{n \rightarrow \infty} \sigma\left(x^{*}, \mathscr{M}_{\alpha_{n}}\left(A_{j}\right)\right) .
\end{array}
$$

This shows the countable additivity of $\lim _{n \rightarrow \infty} \sigma\left(x^{*}, \mathscr{M}_{\alpha_{n}}(\cdot)\right)$ for each $x^{*} \in \mathscr{X}^{*}$.

(2) Suppose that $\mathscr{M}_{\alpha_{n}} \ll \mu$ for each $\alpha_{n} \in(0,1]$. If, for $A \in \mathscr{A}, \mu(A)=0$, then $\mathscr{M}_{\alpha_{n}}(A)=\{0\}$ which implies that $\sigma\left(x^{*}, \mathscr{M}_{\alpha_{n}}(A)\right)=0$ for each $x^{*} \in \mathscr{X}^{*}$. It follows that $\lim _{n \rightarrow \infty} \sigma\left(x^{*}, \mathscr{M}_{\alpha_{n}}(A)\right)=0$ for each $x^{*} \in \mathscr{X}^{*}$. Thus $\lim _{n \rightarrow \infty} \sigma\left(x^{*}, \mathscr{M}_{\alpha_{n}}(\cdot)\right) \ll \mu$ for each $x^{*} \in \mathscr{X}^{*}$.

(3) Suppose that $\mathscr{M}_{\alpha_{n}} \perp \mu$ for each $\alpha_{n} \in(0,1]$. If there exists $N \in \mathscr{A}, \mu(N)=0$, then, for arbitrary $A \in \mathscr{A}, \mathscr{M}_{\alpha_{n}}(A \cap$ $\left.N^{c}\right)=\{0\}$ which implies that $\sigma\left(x^{*}, \mathscr{M}_{\alpha_{n}}\left(A \cap N^{c}\right)\right)=0$ for all $\alpha \in(0,1]$. It follows that $\lim _{n \rightarrow \infty} \sigma\left(x^{*}, \mathscr{M}_{\alpha_{n}}(A)\right)=0$ for each $x^{*} \in \mathscr{X}^{*}$. Thus $\lim _{n \rightarrow \infty} \sigma\left(x^{*}, \mathscr{M}_{\alpha_{n}}(\cdot)\right) \perp \mu$ for each $x^{*} \in$ $x^{*}$.

In the following, we give the Lebesgue type decomposition theorem for generalized fuzzy number measures.

Theorem 11. Let $\widetilde{\mathscr{M}}: \mathscr{A} \rightarrow \mathscr{F}_{w k c}(\mathscr{X})$ be a generalized fuzzy number measure. Then there exists a unique pair of generalized fuzzy number measures $\widetilde{\mathscr{M}}_{c}$ and $\widetilde{\mathscr{M}}_{s}$ such that

$$
\begin{aligned}
& \widetilde{\mathscr{M}}=\widetilde{\mathscr{M}}_{c}+\widetilde{\mathscr{M}}_{s}, \\
& \widetilde{\mathscr{M}}_{c} \ll \mu, \\
& \widetilde{\mathscr{M}}_{s} \perp \mu .
\end{aligned}
$$

Proof. Firstly, we show the existence of decomposition. If $\widetilde{\mathbb{M}}$ : $\mathscr{A} \rightarrow \mathscr{F}_{w k c}(\mathscr{X})$ is a generalized fuzzy number measure, then, by Theorem 6, $\widetilde{M}_{\alpha}: \mathscr{A} \rightarrow \mathscr{P}_{w k c}(\mathscr{X})$ defined by

$$
\widetilde{\mathscr{M}}_{\alpha}(A)=\{x \in \mathscr{X}: \widetilde{\mathscr{M}}(A)(x) \geq \alpha\}
$$

is a set valued measure for each $\alpha \in(0,1]$. It follows from Theorem 7 that there exists a unique pair of weakly compact and convex set valued measures $\widetilde{\mathscr{M}}_{\alpha}^{(c)}$ and $\widetilde{\mathscr{M}}_{\alpha}^{(s)}$ such that

$$
\begin{aligned}
& \widetilde{\mathscr{M}}_{\alpha}=\widetilde{\mathscr{M}}_{\alpha}^{(c)}+\widetilde{\mathscr{M}}_{\alpha}^{(s)}, \\
& \widetilde{\mathscr{M}}_{\alpha}^{(c)} \ll \mu, \\
& \widetilde{\mathscr{M}}_{\alpha}^{(s)} \perp \mu
\end{aligned}
$$

for each $\alpha \in(0,1]$. Further,

$$
\sigma\left(x^{*}, \widetilde{\mathscr{M}}_{\alpha}(\cdot)\right)=\sigma\left(x^{*}, \widetilde{\mathscr{M}}_{\alpha}^{(c)}(\cdot)\right)+\sigma\left(x^{*}, \widetilde{\mathbb{M}}_{\alpha}^{(s)}(\cdot)\right)
$$

is the Lebesgue decomposition of $\sigma\left(x^{*}, \widetilde{\mathscr{M}}_{\alpha}(\cdot)\right)$ for any $x^{*} \in$ $\mathscr{X}^{*}$ and $\alpha \in(0,1]$.

To complete the proof, by Theorem 6 , we show that $\left\{\widetilde{\mathscr{M}}_{\alpha}^{(c)}\right\}_{\alpha \in(0,1]}$ and $\left\{\widetilde{\mathscr{M}}_{\alpha}^{(s)}\right\}_{\alpha \in(0,1]}$ define two generalized fuzzy number measures $\widetilde{\mathscr{M}}_{c}$ and $\widetilde{\mathscr{M}}_{s}$ such that

$$
\begin{aligned}
& \widetilde{\mathscr{M}}=\widetilde{\mathscr{M}}_{c}+\widetilde{\mathscr{M}}_{s}, \\
& \widetilde{\mathscr{M}}_{c} \ll \mu, \\
& \widetilde{\mathscr{M}}_{s} \perp \mu .
\end{aligned}
$$

From (18), we have

$$
\begin{aligned}
\sigma\left(x^{*}, \widetilde{\mathscr{M}}_{\alpha}(A)\right)= & \sigma\left(x^{*}, \widetilde{\mathscr{M}}_{\alpha}^{(c)}(A)\right) \\
& +\sigma\left(x^{*}, \widetilde{\mathscr{M}}_{\alpha}^{(s)}(A)\right)
\end{aligned}
$$

for each $A \in \mathscr{A}, x^{*} \in \mathscr{X}^{*}$, and $\alpha \in(0,1]$. Since $\widetilde{\mathscr{M}}$ : $\mathscr{A} \rightarrow \mathscr{F}_{w k c}(\mathscr{X})$ is a generalized fuzzy number measure, by Theorem 6, for arbitrary $\alpha, \beta \in(0,1]$ with $\alpha \leq \beta$ and $A \in \mathscr{A}$, we have $\widetilde{\mathscr{M}}_{\beta}(A) \subseteq \widetilde{\mathscr{M}}_{\alpha}(A)$. It follows that $\sigma\left(x^{*}, \widetilde{\mathscr{M}}_{\beta}(A)\right) \leq$ $\sigma\left(x^{*}, \widetilde{M}_{\alpha}(A)\right)$ for each $A \in \mathscr{A}$ and $x^{*} \in \mathscr{X}^{*}$ if $\alpha \leq \beta$. According to the uniqueness of Lebesgue decomposition for real valued measures, we have

$$
\begin{aligned}
& \sigma\left(x^{*}, \widetilde{\mathscr{M}}_{\beta}^{(c)}(A)\right) \leq \sigma\left(x^{*}, \widetilde{\mathscr{M}}_{\alpha}^{(c)}(A)\right), \\
& \sigma\left(x^{*}, \widetilde{\mathscr{M}}_{\beta}^{(s)}(A)\right) \leq \sigma\left(x^{*}, \widetilde{\mathscr{M}}_{\alpha}^{(s)}(A)\right)
\end{aligned}
$$

for each $x^{*} \in \mathscr{X}^{*}$ and $A \in \mathscr{A}$, which imply

$$
\begin{aligned}
& \widetilde{\mathscr{M}}_{\beta}^{(c)}(A) \subseteq \widetilde{\mathscr{M}}_{\alpha}^{(c)}(A), \\
& \widetilde{\mathscr{M}}_{\beta}^{(s)}(A) \subseteq \widetilde{\mathscr{M}}_{\alpha}^{(s)}(A)
\end{aligned}
$$

for each $A \in \mathscr{A}$. Now let $\left\{\alpha_{n}\right\}_{n \in \mathbb{N}}$ be a nondecreasing sequence in $[0,1]$ converging to $\alpha \in(0,1]$. We use Theorem 3 to show that

$$
\begin{aligned}
& \widetilde{\mathscr{M}}_{\alpha}^{(c)}(A)=\bigcap_{n=1}^{\infty} \widetilde{\mathscr{M}}_{\alpha_{n}}^{(c)}(A), \\
& \widetilde{\mathscr{M}}_{\alpha}^{(s)}(A)=\bigcap_{n=1}^{\infty} \widetilde{\mathscr{M}}_{\alpha_{n}}^{(s)}(A)
\end{aligned}
$$


for any $A \in \mathscr{A}$. By Theorem 7 , we can conclude that there exists a unique pair of weakly compact and convex set valued measures $\widetilde{\mathscr{M}}_{\alpha_{n}}^{(c)}$ and $\widetilde{\mathscr{M}}_{\alpha_{n}}^{(s)}$ such that

$$
\begin{aligned}
& \widetilde{\mathscr{M}}_{\alpha_{n}}=\widetilde{\mathscr{M}}_{\alpha_{n}}^{(c)}+\widetilde{\mathscr{M}}_{\alpha_{n}}^{(s)}, \\
& \widetilde{\mathscr{M}}_{\alpha_{n}}^{(c)} \ll \mu, \\
& \widetilde{\mathscr{M}}_{\alpha_{n}}^{(s)} \perp \mu
\end{aligned}
$$

for any $\alpha_{n} \in(0,1]$. It follows that

$$
\begin{aligned}
\sigma\left(x^{*}, \widetilde{\mathscr{M}}_{\alpha_{n}}(A)\right)= & \sigma\left(x^{*}, \widetilde{\mathscr{M}}_{\alpha_{n}}^{(c)}(A)+\widetilde{\mathscr{M}}_{\alpha_{n}}^{(s)}(A)\right) \\
= & \sigma\left(x^{*}, \widetilde{\mathscr{M}}_{\alpha_{n}}^{(c)}(A)\right) \\
& +\sigma\left(x^{*}, \widetilde{\mathscr{M}}_{\alpha_{n}}^{(s)}(A)\right)
\end{aligned}
$$

for any $x^{*} \in \mathscr{X}^{*}$ and $A \in \mathscr{A}$. Lemma 10 ensures that

$$
\begin{aligned}
& \lim _{n \rightarrow \infty} \sigma\left(x^{*}, \widetilde{\mathscr{M}}_{\alpha_{n}}^{(c)}(\cdot)\right), \\
& \lim _{n \rightarrow \infty} \sigma\left(x^{*}, \widetilde{\mathscr{M}}_{\alpha_{n}}^{(s)}(\cdot)\right)
\end{aligned}
$$

exist. Then, from (25), we have

$$
\begin{aligned}
& \lim _{n \rightarrow \infty} \sigma\left(x^{*}, \widetilde{\mathscr{M}}_{\alpha_{n}}(A)\right) \\
& \quad=\lim _{n \rightarrow \infty}\left(\sigma\left(x^{*}, \widetilde{\mathscr{M}}_{\alpha_{n}}^{(c)}(A)\right)+\sigma\left(x^{*}, \widetilde{\mathscr{M}}_{\alpha_{n}}^{(s)}(A)\right)\right) \\
& \quad=\lim _{n \rightarrow \infty} \sigma\left(x^{*}, \widetilde{\mathscr{M}}_{\alpha_{n}}^{(c)}(A)\right)+\lim _{n \rightarrow \infty} \sigma\left(x^{*}, \widetilde{\mathscr{M}}_{\alpha_{n}}^{(s)}(A)\right) .
\end{aligned}
$$

Again by Theorem 6, we have

$$
\widetilde{\mathscr{M}}_{\alpha}(A)=\bigcap_{n=1}^{\infty} \widetilde{\mathscr{M}}_{\alpha_{n}}(A)
$$

for any $A \in \mathscr{A}$. It follows from Theorem 3 that

$$
\lim _{n \rightarrow \infty} \sigma\left(x^{*}, \widetilde{\mathscr{M}}_{\alpha_{n}}(A)\right)=\sigma\left(x^{*}, \widetilde{\mathscr{M}}_{\alpha}(A)\right)
$$

for each $x^{*} \in \mathscr{X}^{*}$. Then (27) and (29) imply relation

$$
\begin{aligned}
\sigma\left(x^{*}, \widetilde{M}_{\alpha}(A)\right)= & \lim _{n \rightarrow \infty} \sigma\left(x^{*}, \widetilde{M}_{\alpha_{n}}^{(c)}(A)\right) \\
& +\lim _{n \rightarrow \infty} \sigma\left(x^{*}, \widetilde{M}_{\alpha_{n}}^{(s)}(A)\right)
\end{aligned}
$$

for each $x^{*} \in \mathscr{X}^{*}$ and $A \in \mathscr{A}$. Lemma 10 shows that

$$
\begin{aligned}
& \lim _{n \rightarrow \infty} \sigma\left(x^{*}, \widetilde{\mathscr{M}}_{\alpha_{n}}^{(c)}(\cdot)\right), \\
& \lim _{n \rightarrow \infty} \sigma\left(x^{*}, \widetilde{\mathscr{M}}_{\alpha_{n}}^{(s)}(\cdot)\right)
\end{aligned}
$$

are two real valued measures such that

$$
\begin{aligned}
& \lim _{n \rightarrow \infty} \sigma\left(x^{*}, \widetilde{\mathscr{M}}_{\alpha_{n}}^{(c)}(\cdot)\right) \ll \mu, \\
& \lim _{n \rightarrow \infty} \sigma\left(x^{*}, \widetilde{\mathscr{M}}_{\alpha_{n}}^{(s)}(\cdot)\right) \perp \mu .
\end{aligned}
$$

Then, by uniqueness of Lebesgue decomposition for real valued measure, (20) and (30) imply

$$
\begin{aligned}
& \sigma\left(x^{*}, \widetilde{M}_{\alpha}^{(c)}(A)\right)=\lim _{n \rightarrow \infty} \sigma\left(x^{*}, \widetilde{M}_{\alpha_{n}}^{(c)}(A)\right), \\
& \sigma\left(x^{*}, \widetilde{\mathscr{M}}_{\alpha}^{(s)}(A)\right)=\lim _{n \rightarrow \infty} \sigma\left(x^{*}, \widetilde{\mathscr{M}}_{\alpha_{n}}^{(s)}(A)\right)
\end{aligned}
$$

for any $A \in \mathscr{A}$. It follows from Theorem 3 that

$$
\begin{aligned}
& \widetilde{\mathscr{M}}_{\alpha}^{(c)}(A)=\bigcap_{n=1}^{\infty} \widetilde{\mathscr{M}}_{\alpha_{n}}^{(c)}(A), \\
& \widetilde{\mathscr{M}}_{\alpha}^{(s)}(A)=\bigcap_{n=1}^{\infty} \widetilde{\mathscr{M}}_{\alpha_{n}}^{(s)}(A)
\end{aligned}
$$

for each $A \in \mathscr{A}$. Then $\left\{\widetilde{\mathscr{M}}_{\alpha}^{(c)}(\cdot)\right\}_{\alpha \in(0,1]}$ and $\left\{\widetilde{\mathscr{M}}_{\alpha}^{(s)}(\cdot)\right\}_{\alpha \in(0,1]}$ satisfy conditions (1) and (2) of Theorem 6, respectively. For any $A \in \mathscr{A}$, let

$$
\widetilde{\mathscr{M}}_{i}(A)(x)= \begin{cases}\sup \left\{\alpha \in(0,1]: x \in \widetilde{\mathscr{M}}_{\alpha}^{(i)}(A)\right\}, & \text { if }\left\{\alpha \in(0,1], x \in \widetilde{\mathscr{M}}_{\alpha}^{(i)}(A)\right\} \neq \emptyset \\ 0, & \text { if }\left\{\alpha \in(0,1], x \in \widetilde{\mathscr{M}}_{\alpha}^{(i)}(A)\right\}=\emptyset\end{cases}
$$

where $i=c$, s. Then $\widetilde{\mathscr{M}}_{c}$ and $\widetilde{\mathscr{M}}_{s}$ define two generalized fuzzy number measures such that

$$
\begin{aligned}
& \left(\widetilde{\mathscr{M}}_{c}(A)\right)_{\alpha}=\widetilde{\mathscr{M}}_{\alpha}^{(c)}(A), \\
& \left(\widetilde{\mathscr{M}}_{s}(A)\right)_{\alpha}=\widetilde{\mathscr{M}}_{\alpha}^{(s)}(A)
\end{aligned}
$$

for any $A \in \mathscr{A}$ and $\alpha \in(0,1]$. Since $\widetilde{\mathscr{M}}_{\alpha}^{(c)} \ll \mu, \widetilde{\mathscr{M}}_{\alpha}^{(s)} \perp \mu$ for all $\alpha \in(0,1]$, by Lemma $10, \widetilde{\mathscr{M}}_{c} \ll \mu$ and $\widetilde{\mathscr{M}}_{s} \perp \mu$.
In the following, we claim that $\widetilde{\mathscr{M}}=\widetilde{\mathscr{M}}_{c}+\widetilde{\mathscr{M}}_{s}$. Obviously, for any $A \in \mathscr{A}, \widetilde{\mathscr{M}}_{c}(A)+\widetilde{\mathscr{M}}_{s}(A) \in \mathscr{F}_{w k c}(\mathscr{X})$ and for any $\alpha \in(0,1]$,

$$
\begin{aligned}
\left(\widetilde{\mathscr{M}}_{c}(A)+\widetilde{\mathscr{M}}_{s}(A)\right)_{\alpha} & =\left(\widetilde{\mathscr{M}}_{c}(A)\right)_{\alpha}+\left(\widetilde{\mathscr{M}}_{s}(A)\right)_{\alpha} \\
& =\widetilde{\mathscr{M}}_{\alpha}^{(c)}(A)+\widetilde{\mathscr{M}}_{\alpha}^{(s)}(A) \\
& =\widetilde{\mathscr{M}}_{\alpha}(A) .
\end{aligned}
$$


Then, if $\widetilde{\mathscr{M}}(A)(x)>0$,

$$
\begin{aligned}
\widetilde{\mathscr{M}} & (A)(x)=\sup \left\{\alpha \in(0,1]: x \in \widetilde{\mathscr{M}}_{\alpha}(A)\right\} \\
& =\sup \left\{\alpha \in(0,1]: x \in\left(\widetilde{\mathscr{M}}_{c}(A)+\widetilde{\mathscr{M}}_{s}(A)\right)_{\alpha}\right\} \\
& =\left(\widetilde{\mathscr{M}}_{c}(A)+\widetilde{\mathscr{M}}_{s}(A)\right)(x) .
\end{aligned}
$$

If $\widetilde{M}(A)(x)=0$, then $\left\{\alpha \in(0,1]: x \in \widetilde{M}_{\alpha}(A)\right\}=\emptyset$, which implies that, for any $\alpha \in(0,1], x \notin \widetilde{M}_{\alpha}(A)$. Hence, for any $\alpha \in(0,1], x \notin\left(\widetilde{\mathscr{M}}_{c}(A)+\widetilde{\mathscr{M}}_{s}(A)\right)_{\alpha}$; that is, $\{\alpha \in$ $\left.(0,1]: x \in\left(\widetilde{\mathscr{M}}_{c}(A)+\widetilde{\mathscr{M}}_{s}(A)\right)_{\alpha}\right\}=\emptyset$, which implies that $\left(\widetilde{\mathscr{M}}_{c}(A)+\widetilde{\mathscr{M}}_{s}(A)\right)(x)=0$. It follows that $\widetilde{\mathscr{M}}(A)(x)=$ $\left(\widetilde{\mathscr{M}}_{c}(A)+\widetilde{\mathscr{M}}_{s}(A)\right)(x)$.

Lastly, we show the uniqueness of decomposition. Suppose that there exist generalized fuzzy number valued measures $\widetilde{\mathscr{M}}_{c j}, \widetilde{\mathscr{M}}_{s j}, j=1,2$, such that

$$
\begin{aligned}
& \widetilde{\mathscr{M}}=\widetilde{\mathscr{M}}_{c j}+\widetilde{\mathscr{M}}_{s j}, \\
& \widetilde{\mathscr{M}}_{c j} \ll \mu, \\
& \widetilde{\mathscr{M}}_{s j} \perp \mu,
\end{aligned}
$$

$$
j=1,2 \text {. }
$$

Then, for any $\alpha \in(0,1]$,

$$
\begin{aligned}
& \widetilde{\mathscr{M}}_{\alpha}=\left(\widetilde{\mathscr{M}}_{c 1}\right)_{\alpha}+\left(\widetilde{\mathscr{M}}_{s 1}\right)_{\alpha}, \\
& \left(\widetilde{\mathscr{M}}_{c 1}\right)_{\alpha} \ll \mu, \\
& \left(\widetilde{\mathscr{M}}_{s 1}\right)_{\alpha} \perp \mu, \\
& \widetilde{\mathscr{M}}_{\alpha}=\left(\widetilde{\mathscr{M}}_{c 2}\right)_{\alpha}+\left(\widetilde{\mathscr{M}}_{s 2}\right)_{\alpha}, \\
& \left(\widetilde{\mathscr{M}}_{c 2}\right)_{\alpha} \ll \mu, \\
& \left(\widetilde{\mathscr{M}}_{s 2}\right)_{\alpha} \perp \mu .
\end{aligned}
$$

According to the uniqueness of Lebesgue decomposition of set valued measures, we have

$$
\begin{aligned}
& \left(\widetilde{\mathscr{M}}_{c 1}\right)_{\alpha}=\left(\widetilde{\mathscr{M}}_{c 2}\right)_{\alpha}, \\
& \left(\widetilde{\mathscr{M}}_{s 1}\right)_{\alpha}=\left(\widetilde{\mathscr{M}}_{s 2}\right)_{\alpha},
\end{aligned}
$$

which implies that $\widetilde{\mathscr{M}}_{c 1}=\widetilde{\mathscr{M}}_{c 2}, \widetilde{\mathscr{M}}_{s 1}=\widetilde{\mathscr{M}}_{s 2}$. This completes the proof.

In the following, we generalize weak Radon-Nikodým theorem for set valued measures [20] to generalized fuzzy number measures.

Let $F: \Omega \rightarrow \mathscr{P}_{f}(\mathscr{X})$ be a set valued function. $F(\omega)$ is said to be scalarly measurable if, for each $x^{*} \in \mathscr{X}^{*}$, the real valued function $\sigma\left(x^{*}, F(\omega)\right)$ is measurable. $F: \Omega \rightarrow \mathscr{P}_{f}(\mathscr{X})$ is said to be weakly integrable bounded if the real valued function $\left|x^{*} F\right|: \Omega \rightarrow \mathbb{R}$ defined by $\left|x^{*} F\right|(\omega)=\sup _{x \in F(\omega)}\left|\left\langle x^{*}, x\right\rangle\right|$ is integrable for each $x^{*} \in \mathscr{X}^{*} \cdot f: \Omega \rightarrow \mathscr{X}$ is called a measurable selector of $F: \Omega \rightarrow \mathscr{P}_{f}(\mathscr{X})$ if $f(\omega) \in F(\omega)$ for each $\omega \in \Omega$. A measurable selector $f(\omega)$ of $F(\omega)$ is called a Pettis integrable selector of $F(\omega)$ if $f(\omega)$ is Pettis integrable. We denote by $S_{w F}$ the set of all Pettis integrable selectors of $F(\omega) . F: \Omega \rightarrow \mathscr{P}_{f}(\mathscr{X})$ is said to be Aumann-Pettis integrable if $S_{w F} \neq \emptyset$. In this case, the Aumann-Pettis integral of $F: \Omega \rightarrow \mathscr{P}_{f}(\mathscr{X})$ is defined by

$$
\begin{aligned}
(w) & \int_{A} F(\omega) \mathrm{d} \mu(\omega) \\
= & \left\{(w) \int_{A} f(\omega) \mathrm{d} \mu(\omega): f \in S_{w F}\right\}, \quad \forall A \in \mathscr{A} .
\end{aligned}
$$

We denote by $L_{w k c}^{w}(\Omega, \mathscr{X})$ the set of all Aumann-Pettis integrable, weakly compact, and convex set valued functions.

Theorem 12 (see [20]). Suppose that $\mathscr{X}$ has the RNP and $\mathscr{X}^{*}$ is separable. Let $\mathscr{M}: \mathscr{A} \rightarrow \mathscr{P}_{w k c}(\mathscr{X})$ be a $\mu$-continuous set valued measure of bounded variation. $M$ has a weak RadonNikodým derivative $F \in L_{w k c}^{w}(\Omega, \mathscr{X})$ if and only if $\mathscr{M}$ is of $\sigma$ bounded variation.

The mapping $\widetilde{F}: \Omega \rightarrow \mathscr{F}_{w k c}(\mathscr{X})$ is called a measurable fuzzy mapping, if $\widetilde{F}_{\alpha}(\omega)=\{x \in \mathscr{X}: \widetilde{F}(\omega)(x) \geq \alpha\}$ is a measurable set valued mapping for each $\alpha \in(0,1]$. $\widetilde{F}(\omega)$ is called weakly integrable bounded if $\widetilde{F}_{\alpha}(\omega)$ is weakly integrable bounded set valued mapping for each $\alpha \in(0,1]$.

Definition 13 (see [19]). The mapping $\widetilde{F}: \Omega \rightarrow \mathscr{F}_{w k c}(\mathscr{X})$ is said to be weakly integrable, if, for each $A \in \mathscr{A}$, there exist $\widetilde{u}_{A} \in \mathscr{F}_{w k c}(\mathscr{X})$ such that, for each $\alpha \in(0,1]$,

$$
\left(\widetilde{u}_{A}\right)_{\alpha}=(w) \int_{A} \widetilde{F}_{\alpha}(\omega) \mathrm{d} \mu(\omega)
$$

In this case $\widetilde{u}_{A}=(w) \int_{A} \widetilde{F}(\omega) \mathrm{d} \mu(\omega)$ is called the weak integral of $\widetilde{F}(\omega)$ over $A$.

Let $\mathscr{L}_{w k c}^{w}(\Omega, \mathscr{X})$ denote the set of all weakly integrable bounded measurable fuzzy mappings.

Definition 14. Let $\widetilde{\mathscr{M}}: \mathscr{A} \rightarrow \mathscr{F}_{w k c}(\mathscr{X})$ be a generalized fuzzy number measure. If there exists a weakly integrable bounded measurable fuzzy mapping $\widetilde{F}: \Omega \rightarrow \mathscr{F}_{w k c}(\mathscr{X})$ such that

$$
\widetilde{\mathscr{M}}(A)=(w) \int_{A} \widetilde{F}(\omega) \mathrm{d} \mu(\omega), \quad \forall A \in \mathscr{A},
$$

then one calls that $\widetilde{F}: \Omega \rightarrow \mathscr{F}_{w k c}(\mathscr{X})$ is a weak RadonNikodým derivative for $\widetilde{\mathscr{M}}$ with respect to $\mu$.

We call that $\widetilde{\mathscr{M}}$ is of $\sigma$-bounded variation if $\widetilde{\mathscr{M}}_{\alpha}$ is of $\sigma$ bounded variation for each $\alpha \in(0,1]$. In the following we show the existence of weak Radon-Nikodým derivatives for generalized fuzzy number measures.

Theorem 15. Suppose that $\mathscr{X}$ has the RNP and $\mathscr{X}^{*}$ is separable. Let $\widetilde{\mathscr{M}}: \mathscr{A} \rightarrow \mathscr{F}_{w k c}(\mathscr{X})$ be a $\mu$-continuous generalized fuzzy number measure of $\sigma$-bounded variation. Then $\widetilde{M}$ has a weak Radon-Nikodým derivative $\widetilde{F} \in \mathscr{L}_{w k c}^{w}(\Omega, \mathscr{X})$; namely, 
there exists a weakly integrable bounded measurable fuzzy mapping $\widetilde{F}: \Omega \rightarrow \mathscr{F}_{w k c}(\mathscr{X})$ such that

$$
\widetilde{\mathscr{M}}(A)=(w) \int_{A} \widetilde{F}(\omega) \mathrm{d} \mu(\omega)
$$

for each $A \in \mathscr{A}$.

Proof. Since $\widetilde{\mathscr{M}}: \mathscr{A} \rightarrow \mathscr{F}_{w k c}(\mathscr{X})$ is a generalized fuzzy number measure, by Theorem $6, \widetilde{M}_{\alpha}: \mathscr{A} \rightarrow \mathscr{P}_{w k c}(\mathscr{X})$ defined by

$$
\widetilde{\mathscr{M}}_{\alpha}(A)=\{x \in \mathscr{X}: \widetilde{\mathscr{M}}(A)(x) \geq \alpha\}
$$

is a set valued measure for each $\alpha \in(0,1]$. Since $\widetilde{\mathscr{M}}: \mathscr{A} \rightarrow$ $\mathscr{F}_{w k c}(\mathscr{X})$ is $\mu$-continuous and is of $\sigma$-bounded variation, $\widetilde{M}_{\alpha}: \mathscr{A} \rightarrow \mathscr{P}_{w k c}(\mathscr{X})$ is $\mu$-continuous and is of $\sigma$-bounded variation for each $\alpha \in(0,1]$. According to Theorem 12, for each $\alpha \in(0,1]$ there exists $F_{\alpha} \in L_{w k c}^{w}(\Omega, \mathscr{X})$ which is a weak Radon-Nikodým derivative for $\widetilde{\mathscr{M}}_{\alpha}$ with respect to $\mu$; namely, $F_{\alpha}$ satisfies

$$
\widetilde{\mathscr{M}}_{\alpha}(A)=(w) \int_{A} F_{\alpha}(\omega) \mathrm{d} \mu(\omega)
$$

for each $\alpha \in(0,1]$ and $A \in \mathscr{A}$.

In the following, we will show that the family $\left\{F_{\alpha}(\omega)\right\}_{\alpha \in(0,1]}$ defines a weakly integrable bounded measurable fuzzy mapping $\widetilde{F}(\omega)$. Firstly, we prove that the family $\left\{F_{\alpha}(\omega)\right\}_{\alpha \in(0,1]}$ defines a generalized fuzzy number $\widetilde{F}(\omega)$ for each $\omega \in \Omega$. We will prove that all the conditions of Theorem 2 are satisfied. Obviously, $F_{\alpha}(\omega) \in \mathscr{P}_{w k c}(\mathscr{X})$ for all $\alpha \in(0,1]$ and $\omega \in \Omega$. For $0<\beta \leq \alpha \leq 1$, we have $\widetilde{\mathscr{M}}_{\alpha}(A) \subseteq \widetilde{\mathscr{M}}_{\beta}(A)$ for any $A \in \mathscr{A}$ which implies that

$$
(w) \int_{A} F_{\alpha}(\omega) \mathrm{d} \mu(\omega) \subseteq(w) \int_{A} F_{\beta}(\omega) \mathrm{d} \mu(\omega) .
$$

Then, by properties of support function, we have

$$
\begin{aligned}
& \sigma\left(x^{*},(w) \int_{A} F_{\alpha}(\omega) \mathrm{d} \mu(\omega)\right) \\
& \quad \leq \sigma\left(x^{*},(w) \int_{A} F_{\beta}(\omega) \mathrm{d} \mu(\omega)\right)
\end{aligned}
$$

for each $x^{*} \in \mathscr{X}^{*}$. It follows from Lemma 4.3 [19] that

$$
\int_{A} \sigma\left(x^{*}, F_{\alpha}(\omega)\right) \mathrm{d} \mu(\omega) \leq \int_{A} \sigma\left(x^{*}, F_{\beta}(\omega)\right) \mathrm{d} \mu(\omega)
$$

for each $x^{*} \in \mathscr{X}^{*}$. Since this is true for all $A \in \mathscr{A}$ we deduce that

$$
\sigma\left(x^{*}, F_{\alpha}(\omega)\right) \leq \sigma\left(x^{*}, F_{\beta}(\omega)\right) .
$$

Also, since $F_{\alpha}(\omega)$ and $F_{\beta}(\omega)$ are weakly compact and convex, we have $F_{\alpha}(\omega) \subseteq F_{\beta}(\omega)$. Let $\left\{\alpha_{n}\right\}_{n \in \mathbb{N}} \subseteq[0,1]$ be a nondecreasing sequence converging to $\alpha \in(0,1]$. By Theorem 6 , we have $\widetilde{\mathscr{M}}_{\alpha}(A)=\bigcap_{n=1}^{\infty} \widetilde{\mathscr{M}}_{\alpha_{n}}(A)$ which implies that

$$
(w) \int_{A} F_{\alpha}(\omega) \mathrm{d} \mu(\omega)=\bigcap_{n=1}^{\infty}(w) \int_{A} F_{\alpha_{n}}(\omega) \mathrm{d} \mu(\omega)
$$

for each $A \in \mathscr{A}$. It follows from Theorem 3 that

$$
\begin{array}{r}
\lim _{n \rightarrow \infty} \sigma\left(x^{*},(w) \int_{A} F_{\alpha_{n}}(\omega) \mathrm{d} \mu(\omega)\right) \\
=\sigma\left(x^{*},(w) \int_{A} F_{\alpha}(\omega) \mathrm{d} \mu(\omega)\right) .
\end{array}
$$

By Lemma 4.3 [19], we have

$$
\begin{array}{r}
\lim _{n \rightarrow \infty} \int_{A} \sigma\left(x^{*}, F_{\alpha_{n}}(\omega)\right) \mathrm{d} \mu(\omega) \\
=\int_{A} \sigma\left(x^{*}, F_{\alpha}(\omega)\right) \mathrm{d} \mu(\omega) .
\end{array}
$$

Since $F_{\alpha_{n}}(\omega)$ is weak integrable bounded and

$$
\left|\sigma_{F_{\alpha_{n}}(\omega)}\left(x^{*}\right)\right| \leq\left|x^{*} F_{\alpha_{1}}\right|(\omega)
$$

for all $n \in \mathbb{N}, \omega \in \Omega$, and $x^{*} \in \mathscr{X}^{*}$, by Lebesgue's dominated convergence theorem and (54), we obtain that

$$
\begin{aligned}
\lim _{n \rightarrow \infty} & \int_{A} \sigma\left(x^{*}, F_{\alpha_{n}}(\omega)\right) \mathrm{d} \mu(\omega) \\
= & \int_{A} \lim _{n \rightarrow \infty} \sigma\left(x^{*}, F_{\alpha_{n}}(\omega)\right) \mathrm{d} \mu(\omega) \\
= & \int_{A} \sigma\left(x^{*}, F_{\alpha}(\omega)\right) \mathrm{d} \mu(\omega)
\end{aligned}
$$

which implies that

$$
\lim _{n \rightarrow \infty} \sigma\left(x^{*}, F_{\alpha_{n}}(\omega)\right)=\sigma\left(x^{*}, F_{\alpha}(\omega)\right) .
$$

Again, by Theorem 3, we have $\sigma\left(x^{*}, F_{\alpha}(\omega)\right)=$ $\bigcap_{n=1}^{\infty} \sigma\left(x^{*}, F_{\alpha_{n}}(\omega)\right)$. Now, applying Theorem 2 , we get that the family $\left\{F_{\alpha}(\omega)\right\}_{\alpha \in(0,1]}$ generates the unique generalized fuzzy number $\widetilde{F}(\omega)$ such that $\widetilde{F}_{\alpha}(\omega)=F_{\alpha}(\omega)$ for each $\omega \in \Omega$. Since $F_{\alpha}(\omega)$ is weakly integrable bounded set valued measurable function for each $\alpha \in(0,1], \widetilde{F}(\omega)$ is a weakly integrable bounded measurable fuzzy valued function.

To prove that $\widetilde{F}(\omega)$ is a weak Radon-Nikodým derivative for $\widetilde{\mathscr{M}}$ with respect to $\mu$, we will show that

$$
\widetilde{\mathscr{M}}(A)=(w) \int_{A} \widetilde{F}(\omega) \mathrm{d} \mu(\omega)
$$

for each $A \in \mathscr{A}$. By Definition 13,

$$
(w) \int_{A} \widetilde{F}(\omega) \mathrm{d} \mu(\omega) \in \mathscr{F}_{w k c}(\mathscr{X})
$$

such that

$$
\left((w) \int_{A} \widetilde{F}(\omega) \mathrm{d} \mu(\omega)\right)_{\alpha}=(w) \int_{A} \widetilde{F}_{\alpha}(\omega) \mathrm{d} \mu(\omega)
$$

for each $\alpha \in(0,1]$ and $A \in \mathscr{A}$. Together with (47), we have

$$
\begin{aligned}
& \left((w) \int_{A} \widetilde{F}(\omega) \mathrm{d} \mu(\omega)\right)_{\alpha}=(w) \int_{A} \widetilde{F}_{\alpha}(\omega) \mathrm{d} \mu(\omega) \\
& =(w) \int_{A} F_{\alpha}(\omega) \mathrm{d} \mu(\omega)=\widetilde{\mathscr{M}}_{\alpha}(A)=(\widetilde{\mathscr{M}}(A))_{\alpha}
\end{aligned}
$$


for each $\alpha \in(0,1]$ and $A \in \mathscr{A}$, which implies that

$$
\widetilde{\mathscr{M}}(A)=(w) \int_{A} \widetilde{F}(\omega) \mathrm{d} \mu(\omega)
$$

for each $A \in \mathscr{A}$. This completes the proof.

\section{Conflict of Interests}

The authors declare that there is no conflict of interests regarding the publication of this paper.

\section{Acknowledgments}

The project is supported by the National Natural Science Foundation of China (11371002, 41201327, and 61572011), Youth Scientific Research Foundation of Education Department of Hebei Province (QN2015005, QN2015026, and QN20131055), Specialized Research Fund for the Doctoral Program of Higher Education (20131101110048), Natural Science Foundation of Hebei Province (A2013201119), and Special Fund for Enhancing Comprehensive Strength of Midwest China.

\section{References}

[1] Z. Artstein, "Set-valued measures," Transactions of the American Mathematical Society, vol. 165, pp. 103-125, 1972.

[2] B. Cascales, V. Kadets, and J. Rodríguez, "Measurable selectors and set-valued Pettis integral in non-separable Banach spaces," Journal of Functional Analysis, vol. 256, no. 3, pp. 673-699, 2009.

[3] B. Cascales, V. Kadets, and J. Rodríguez, "Radon-Nikodým theorems for multimeasures in non-separable spaces," Journal of Mathematical Physics, Analysis, Geometry, vol. 9, no. 1, pp. 724, 2013.

[4] F. Hiai, "Radon-Nikodym theorems for set-valued measures," Journal of Multivariate Analysis, vol. 8, no. 1, pp. 96-118, 1978.

[5] N. S. Papageorgiou, "Contributions to the theory of set valued functions and set valued measures," Transactions of the American Mathematical Society, vol. 304, no. 1, pp. 245-245, 1987.

[6] M. Stojaković, "Set valued probability and its connection with set valued measure," Statistics \& Probability Letters, vol. 82, no. 6, pp. 1043-1048, 2012.

[7] W. Zhang, S. Li, Z. Wang, and Y. Gao, Set-Valued Stochastic Process, Academic Press, Beijing, China, 2007.

[8] C.-L. Zhou and F.-G. Shi, "New set-valued integral in a Banach space," Journal of Function Spaces, vol. 2015, Article ID 260238, 8 pages, 2015.

[9] M. L. Puri and D. A. Ralescu, "Convergence theorem for fuzzy martingales," Journal of Mathematical Analysis and Applications, vol. 160, no. 1, pp. 107-122, 1991.

[10] J. Bán, "Radon-Nikodým theorem and conditional expectation of fuzzy-valued measures and variables," Fuzzy Sets and Systems, vol. 34, no. 3, pp. 383-392, 1990.

[11] M. Stojaković, "Decomposition and representation of fuzzy valued measure," Fuzzy Sets and Systems, vol. 112, no. 2, pp. 251256, 2000.

[12] M. Stojaković, "Imprecise set and fuzzy valued probability," Journal of Computational and Applied Mathematics, vol. 235, no. 16, pp. 4524-4531, 2011.
[13] M. Stojaković and Z. Stojaković, "Integral with respect to fuzzy measure in finite dimensional Banach spaces," Novi Sad Journal of Mathematics, vol. 37, no. 1, pp. 163-170, 2007.

[14] M. Stojaković and Z. Stojaković, "Fuzzy valued measure based integral," Novi Sad Journal of Mathematics, vol. 37, no. 2, pp. 207220, 2007.

[15] X. Xue, M. Ha, and C. Wu, "On the extension of the fuzzy number measures in Banach spaces: part I. Representation of the fuzzy number measures," Fuzzy Sets and Systems, vol. 78, no. 3, pp. 347-354, 1996.

[16] C.-K. Park, "Generalized fuzzy number valued Bartle integrals," Communications of the Korean Mathematical Society, vol. 25, no. 1, pp. 37-49, 2010.

[17] J. Wu, X. Xue, and C. Wu, "Radon-Nikodym theorem and VitaliHahn-Saks theorem on fuzzy number measures in Banach spaces," Fuzzy Sets and Systems, vol. 117, no. 3, pp. 339-346, 2001.

[18] J. Wu and C. Wu, "The w-derivatives of fuzzy mappings in Banach spaces," Fuzzy Sets and Systems, vol. 119, no. 3, pp. 375381, 2001.

[19] X. P. Xue, M. H. Ha, and M. Ma, "Random fuzzy number integrals in Banach spaces," Fuzzy Sets and Systems, vol. 66, no. 1, pp. 97-111, 1994.

[20] J. R. Wu and C. X. Wu, "Existence of weak Radon-Nikodým derivatives for set-valued measures and conditional expectations for weak set-valued random variables," Acta Mathematicae Applicatae Sinica, vol. 24, no. 2, pp. 255-261, 2001. 


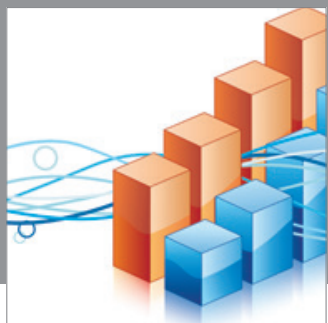

Advances in

Operations Research

mansans

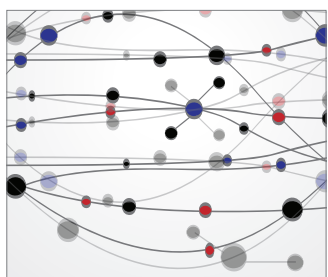

The Scientific World Journal
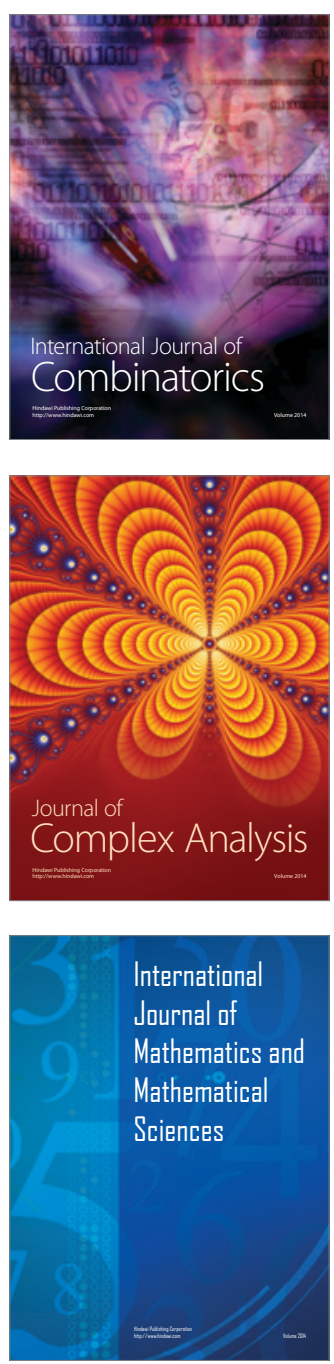
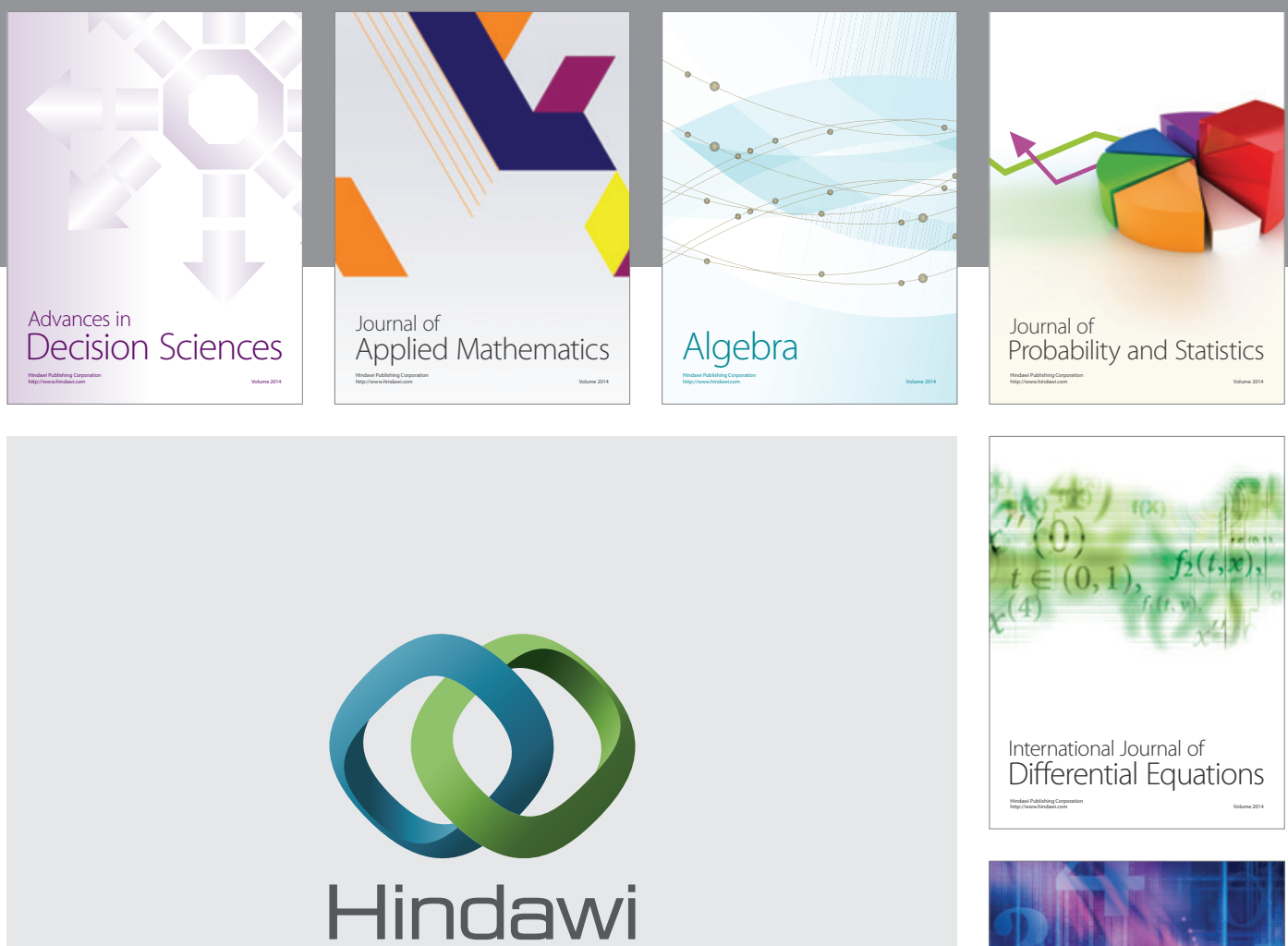

Submit your manuscripts at http://www.hindawi.com
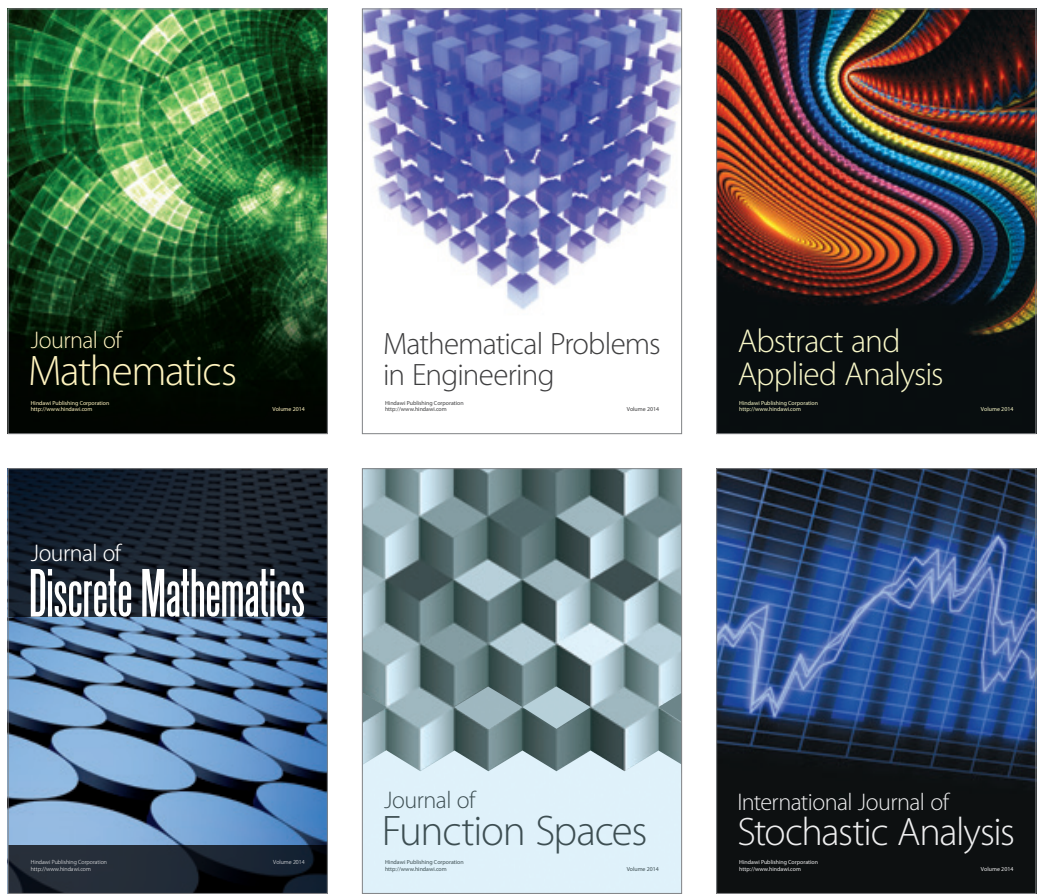

Journal of

Function Spaces

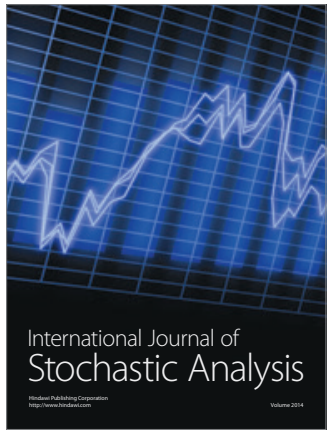

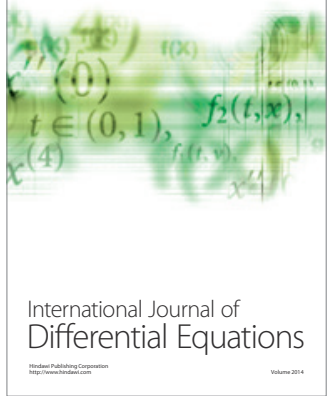
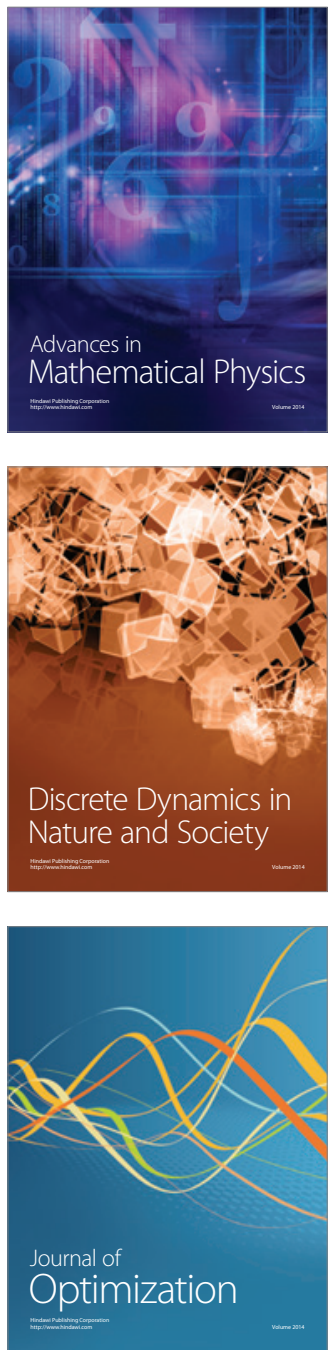